\title{
DISCRETE SEMI-CLASSICAL ORTHOGONAL POLYNOMIALS OF CLASS ONE ON QUADRATIC LATTICES
}

\author{
G. FILIPUK AND M.N. REBOCHO
}

\begin{abstract}
We study orthogonal polynomials on quadratic lattices with respect to a Stieltjes function, $S$, that satisfies a difference equation $A \mathbb{D} S=C \mathbb{M} S+D$, where $A$ is a polynomial of degree less or equal than 3 and $C$ is a polynomial of degree greater or equal than 1 and less or equal than 2 . We show systems of difference equations for the orthogonal polynomials that arise from the so-called compatibility conditions. Some closed formulae for the recurrence relation coefficients are obtained.

KEYWORDS: Discrete orthogonal polynomials; quadratic lattice; divided-difference operator; semi-classical class.

Math. Subject Classification (2010): 33C45, 33C47, 42C05.
\end{abstract}

\section{Introduction}

Discrete semi-classical orthogonal polynomials have been widely studied in the literature of special functions $[12,19,20]$. They are defined through a difference equation with polynomial coefficients for the corresponding Stieltjes function,

$$
A \mathbb{D} S=C \mathbb{M} S+D
$$

Here, $\mathbb{D}$ is some divided-difference operator and $\mathbb{M}$ is a companion difference operator related to $\mathbb{D}$. The divided-difference calculus is classified in terms of hierarchies of operators and related lattices (see, for instance, [22, Sec. 2,3]). In this paper we shall consider the divided-difference operator $\mathbb{D}$ given by

$$
\mathbb{D} f(x(s))=\frac{f(x(s+1 / 2))-f(x(s-1 / 2))}{x(s+1 / 2)-x(s-1 / 2)},
$$

with the so-called quadratic lattice, $x(s)=c_{2} s^{2}+c_{1} s+c_{0}[16$, Sec. 2] (see Section 2 for details). In the literature, these lattices are part of the lattices usually referred to as non-uniform. The calculus on non-uniform lattices

Received November 07, 2018.

The work of M.N. Rebocho is partially supported by the Centre for Mathematics of the University of Coimbra - UID/MAT/00324/2013, funded by the Portuguese Government through FCT/MCTES and co-funded by the European Regional Development Fund through the Partnership Agreement PT2020. 
generalizes the calculus on lattices of lower complexity, such as the linear and $q$-uniform lattices.

There are many papers on semi-classical orthogonal polynomials on quadratic lattices. We refer the interested reader to [7, 8, 11, 22] and their list of references. Standard research topics include the study of structure relations, that is, difference equations involving the polynomials, and systematic classifications or characterizations, given pairs of $(A, C)$ in $(1)$.

In the present paper our goal is twofold. First, to gather some recent results on semi-classical orthogonal polynomials on quadratic lattices, namely, difference equations involving the polynomials and related functions, compatibility relations, and new matrix identities. Essentially, such equations generalize well-known differential systems from [14] (see Section 3). Then, with the help of these results, to describe the sequences of orthogonal polynomials within the class one, that is, under the restrictions $\operatorname{deg}(A) \leq 3,1 \leq \operatorname{deg}(C) \leq 2$ in (1) (see Section 4). The main results are difference equations for the recurrence relation coefficients of the orthogonal polynomials. For the case $\operatorname{deg}(A) \leq 2, \operatorname{deg}(C)=1$, we recover closed form formulae for the classical orthogonal polynomials.

Let us emphasize that, for some lattices of lower complexity, the description of class one has been carried out. For instance, [2] gives the classification and integral representation of semi-classical linear functionals of class one when $\mathbb{D}$ is the derivative operator; in [17], the authors established the system satisfied by the recurrence relation coefficients of symmetric semi-classical orthogonal polynomials of class one when $\mathbb{D}$ is the Hahn's difference operator. We also note [5], an extensive study on semi-classical orthogonal polynomials of class one when $\mathbb{D}$ is the forward difference operator.

The remainder of the paper is organized as follows. In Section 2 we give the definitions and state the basic results which will be used in the forthcoming sections. In Section 3 we show difference equations for semi-classical orthogonal polynomials on quadratic lattices, together with the consequent compatibility relations and matrix identities. In Section 4 we deduce difference equations for the recurrence relation coefficients of the semi-classical orthogonal polynomials. Section 5 is devoted to examples: we show applications on the Dual Hahn polynomials as well as on some of their modifications. 


\section{Divided-difference calculus on quadratic lattices and orthogonal polynomials}

Quadratic lattices are commonly defined through a parametric representation $x=x(s), s \in \mathbf{Z}$,

$$
x(s)=\check{c_{2}} s^{2}+\check{c_{1}} s+\check{c_{0}},
$$

for appropriate constants $\check{c_{j}}$ 's [19, 20]. The corresponding divided-difference operator, defined on the space of arbitrary functions, is given by $[1,18,19]$

$$
\mathbb{D} f(x(s))=\frac{f(x(s+1 / 2))-f(x(s-1 / 2))}{x(s+1 / 2)-x(s-1 / 2)} .
$$

Alternatively, $\mathbb{D}$ can be defined in terms of two functions, say $y_{+}, y_{-}$, as $[15,22]$

$$
(\mathbb{D} f)(x)=\frac{f\left(y_{+}(x)\right)-f\left(y_{-}(x)\right)}{y_{+}(x)-y_{-}(x)},
$$

where $y_{-}$and $y_{+}$are the two $y$-roots of a quadratic equation

$$
\hat{a} y^{2}+2 \hat{b} x y+\hat{c} x^{2}+2 \hat{d} y+2 \hat{e} x+\hat{f}=0, \quad \hat{a} \hat{c} \neq 0, \quad \hat{b}^{2}=\hat{a} \hat{c} .
$$

As $y_{-}, y_{+}$are the $y$-roots of (4), we have

$$
y_{-}(x)=p(x)-\sqrt{r(x)}, \quad y_{+}(x)=p(x)+\sqrt{r(x)},
$$

with $p, r$ polynomials of degree one (in $x$ ) given by

$$
p(x)=-\frac{\hat{b} x+\hat{d}}{\hat{a}}, \quad r(x)=\frac{2(\hat{b} \hat{d}-\hat{a} \hat{e})}{\hat{a}^{2}} x+\frac{\hat{d}^{2}-\hat{a} \hat{f}}{\hat{a}^{2}} .
$$

The polynomials $p, r$ defined in (6) will play an important role in the sequel. In the account of $(5)$ and $y_{-}(x)=x(s-1 / 2), y_{+}(x)=x(s+1 / 2)$, we have $x(s+1 / 2)+x(s-1 / 2)=2 p(x(s)),(x(s+1 / 2)-x(s-1 / 2))^{2}=4 r(x(s))$.

We take $\Delta_{y}=y_{+}-y_{-}$. From (5), there follows

$$
\Delta_{y}=2 \sqrt{r} \text {. }
$$

Define the operators $\mathbb{E}^{+}$and $\mathbb{E}^{-}$(see [15]), acting on arbitrary functions $f$, as

$$
\mathbb{E}^{ \pm} f(x)=f\left(y_{ \pm}(x)\right)
$$

With this notation, (3) is given by

$$
(\mathbb{D} f)(x)=\frac{\mathbb{E}^{+} f-\mathbb{E}^{-} f}{\mathbb{E}^{+} x-\mathbb{E}^{-} x}
$$


The companion operator of $\mathbb{D}$ is defined as (see [15])

$$
(\mathbb{M} f)(x)=\frac{\mathbb{E}^{+} f(x)+\mathbb{E}^{-} f(x)}{2} .
$$

Note that $\mathbb{D}$ has the following property: if $f(x)$ is a polynomial of degree $n$ in $x$, then $\mathbb{D} f(x)$ is a polynomial of degree $n-1$ in $x . \mathbb{M} f$ is a polynomial whenever $f$ is a polynomial. Furthermore, if $\operatorname{deg}(f)=n$, then $\operatorname{deg}(\mathbb{M} f)=n$.

We emphasize that, throughout the paper, we will deal with polynomials of the variable $x$, not displaying the parametrization (2).

Let us introduce some notations within the functional approach. We take a linear functional, $L: \mathbb{C}[x] \longrightarrow \mathbb{C}$, defined by its moments $\left(u_{n}\right)_{n \geq 0}$,

$$
L\left[x^{n}\right]=u_{n}, \quad n=0,1, \ldots,
$$

under the condition

$$
\operatorname{det}\left[u_{i+j}\right]_{i, j=0}^{n} \neq 0, \quad n \geq 0 .
$$

We shall consider systems of orthogonal polynomials, $\left\{P_{n}\right\}_{n \geq 0}$, with respect to $L$, that is,

$$
L\left[P_{n} P_{m}\right]=h_{n} \delta_{n, m}, \quad n, m=0,1, \ldots,
$$

where $h_{n} \neq 0$ and $\delta_{n, m}$ is the Kronecker's delta. It is well known that (10) is a necessary and sufficient condition for the existence of a sequence of orthogonal polynomials with respect to $L[21]$. Furthermore, if $\operatorname{det}\left[u_{i+j}\right]_{i, j=0}^{n}>0, n \geq$ 0 , then there exists a positive measure $\mu$ such that

$$
L[P]=\int_{\text {supp } \mu} P(x) d \mu(x), \quad \forall P \in \mathbb{C}[x],
$$

thus the family $\left\{P_{n}\right\}_{n \geq 0}$ is said to be orthogonal with respect to $\mu$.

Closely related to $\mathcal{L}$ is the moment generating function, the (formal) Stieltjes function, defined by

$$
S(x)=\sum_{n=0}^{+\infty} u_{n} x^{-n-1} .
$$

Throughout this paper the orthogonal polynomials $P_{n}$ are taken to be monic, $P_{n}(x)=x^{n}+$ lower degree terms, $n \geq 0$, and the sequence $\left\{P_{n}\right\}_{n \geq 0}$ will be denoted by SMOP.

Monic orthogonal polynomials satisfy a three-term recurrence relation [21]

$$
P_{n+1}(x)=\left(x-\beta_{n}\right) P_{n}(x)-\gamma_{n} P_{n-1}(x), \quad n=0,1,2, \ldots,
$$


with $P_{-1}(x)=0, P_{0}(x)=1$, and $\gamma_{n} \neq 0, n \geq 1, \gamma_{0}=1$. The parameters $\beta_{n}, \gamma_{n}$ are the so-called recurrence relation coefficients.

Another relevant sequence, related to $\left\{P_{n}\right\}_{n \geq 0}$, is the sequence of associated polynomials of the first kind, denoted by $\left\{P_{n}^{(1)}\right\}_{n \geq 0}$, defined through the three term recurrence relation

$$
P_{n}^{(1)}(x)=\left(x-\beta_{n}\right) P_{n-1}^{(1)}(x)-\gamma_{n} P_{n-2}^{(1)}(x), \quad n=1,2, \ldots,
$$

with $P_{-1}^{(1)}(x)=0, P_{0}^{(1)}(x)=1$.

The sequence of functions of the second kind, $\left\{q_{n}\right\}_{n \geq 0}$, is defined by

$$
q_{n}(x)=S(x) P_{n}(x)-P_{n-1}^{(1)}(x), \quad n \geq 0,
$$

subject to the initial conditions $q_{-1}(x)=1, q_{0}(x)=S(x)$. It satisfies a three term recurrence relation,

$$
q_{n+1}(x)=\left(x-\beta_{n}\right) q_{n}(x)-\gamma_{n} q_{n-1}(x), \quad n=0,1,2, \ldots .
$$

\section{Semi-classical orthogonal polynomials on quadratic lattices}

Semi-classical orthogonal polynomials on quadratic lattices may be defined through:

(i) a Pearson equation for the linear functional $[9,10]$,

$$
\mathbb{D}(\phi L)=\mathbb{M}(\psi L), \quad \phi \neq 0, \operatorname{deg}(\psi) \geq 1 ;
$$

(ii) a difference equation for the Stieltjes function [15, 22],

$$
A \mathbb{D} S=C \mathbb{M} S+D,
$$

with $A, C, D$ irreducible polynomials (in $x$ );

(iii) a Pearson equation for the weight [3, 22],

$$
A \mathbb{D} w=C \mathbb{M} w .
$$

The polynomials in (17)-(19) are related via $[9,10]$

$$
A=\mathbb{M} \phi-r(x) \mathbb{D} \psi-U_{1} \mathbb{M} \psi, \quad C=-\mathbb{D} \phi+\mathbb{M} \psi+U_{1} \mathbb{D} \psi,
$$

with $U_{1}=\check{c}_{2} / 2$, being $\check{c}_{2}$ defined by (2) (cf. [9, eq. (16)]), thus, in the account of $(7), U_{1}=2 p_{0}$. $D$ is a polynomial depending on $A, C$.

The polynomials $A, C, D$ in (18) satisfy, in the account of (3), (9), and (12),

$$
\operatorname{deg}(A) \leq m+2, \operatorname{deg}(C) \leq m+1, \operatorname{deg}(D) \leq m,
$$


where $m$ is some nonnegative integer. When $m=0$ we get the so-called classical polynomials $[9,18]$.

The class of a linear functional $L$ on quadratic lattices was defined in [10], as the non-negative integer given by

$$
\begin{gathered}
c l(L)=\min _{(f, g) \in \mathcal{X}}\{\max (\operatorname{deg}(f)-2, \operatorname{deg}(g)-1)\}, \\
\mathcal{X}=\left\{(f, g) \in C[x]^{2}: \operatorname{deg}(g) \geq 1 \text { and } \mathbb{D}(f L)=\mathbb{M}(g L)\right\} .
\end{gathered}
$$

In what follows we show some fundamental identities for semi-classical orthogonal polynomials on quadratic lattices.

3.1. The system of difference equations for the polynomials. Let $S$ be a Stieltjes function satisfying the difference equation (18), $A \mathbb{D} S=C \mathbb{M} S+D$. Following the same lines as in [22] or [4] (where we take $B \equiv 0$ in Theorem 1 ), we have, for all $n \geq 1$,

$$
\left\{\begin{array}{l}
A \mathbb{D} P_{n}=\left(l_{n-1}+\Delta_{y} \pi_{n-1}\right) \mathbb{E}^{-} P_{n}-C / 2 \mathbb{E}^{+} P_{n}+\Theta_{n-1} \mathbb{E}^{-} P_{n-1}, \\
A \mathbb{D} P_{n-1}^{(1)}=\left(l_{n-1}+\Delta_{y} \pi_{n-1}\right) \mathbb{E}^{-} P_{n-1}^{(1)}+C / 2 \mathbb{E}^{+} P_{n-1}^{(1)}+D \mathbb{E}^{+} P_{n}+\Theta_{n-1} \mathbb{E}^{-} P_{n-2}^{(1)},
\end{array}\right.
$$

and, for all $n \geq 0$,

$$
A \mathbb{D} q_{n}=\left(l_{n-1}+\Delta_{y} \pi_{n-1}\right) \mathbb{E}^{-} q_{n}+C / 2 \mathbb{E}^{+} q_{n}+\Theta_{n-1} \mathbb{E}^{-} q_{n-1} .
$$

The above difference equations (22) are equivalent to

$$
\left\{\begin{array}{l}
A \mathbb{D} P_{n}=\left(l_{n-1}-\Delta_{y} \pi_{n-1}\right) \mathbb{E}^{+} P_{n}-C / 2 \mathbb{E}^{-} P_{n}+\Theta_{n-1} \mathbb{E}^{+} P_{n-1}, \\
A \mathbb{D} P_{n-1}^{(1)}=\left(l_{n-1}-\Delta_{y} \pi_{n-1}\right) \mathbb{E}^{+} P_{n-1}^{(1)}+C / 2 \mathbb{E}^{-} P_{n-1}^{(1)}+D \mathbb{E}^{-} P_{n}+\Theta_{n-1} \mathbb{E}^{+} P_{n-2}^{(1)},
\end{array}\right.
$$

and (23) is equivalent to

$$
A \mathbb{D} q_{n}=\left(l_{n-1}-\Delta_{y} \pi_{n-1}\right) \mathbb{E}^{+} q_{n}+C / 2 \mathbb{E}^{-} q_{n}+\Theta_{n-1} \mathbb{E}^{+} q_{n-1} .
$$

Remark . Furthermore, the polynomials $l_{n}, \Theta_{n}, \pi_{n}$ are subject to the following bounds:

$$
\begin{gathered}
\operatorname{deg}\left(\Theta_{n}\right) \leq \max \{\operatorname{deg}(A)-2, \operatorname{deg}(C)-1\}, \\
\operatorname{deg}\left(l_{n}\right) \leq \max \{\operatorname{deg}(A)-1, \operatorname{deg}(C)\}, \quad \operatorname{deg}\left(\pi_{n}\right) \leq \operatorname{deg}(C)-1 .
\end{gathered}
$$


3.2. Compatibility conditions. Define the matrices

$$
\mathcal{P}_{n}=\left[\begin{array}{cc}
P_{n+1} & P_{n}^{(1)} \\
P_{n} & P_{n-1}^{(1)}
\end{array}\right], \quad n \geq 0 .
$$

In the account of (13) and (14), $\mathcal{P}_{n}$ satisfies the difference equation

$$
\mathcal{P}_{n}=\mathcal{A}_{n} \mathcal{P}_{n-1}, \quad \mathcal{A}_{n}=\left[\begin{array}{cc}
x-\beta_{n} & -\gamma_{n} \\
1 & 0
\end{array}\right], \quad n \geq 1
$$

with initial condition $\mathcal{P}_{0}=\left[\begin{array}{cc}x-\beta_{0} & 1 \\ 1 & 0\end{array}\right]$.

The previous systems (22) and (24) can be put in the matrix form as [7]

$$
\begin{aligned}
& A \mathbb{D} \mathcal{P}_{n}=\mathcal{B}_{n}^{-} \mathbb{E}^{-} \mathcal{P}_{n}-\left(\mathbb{E}^{+} \mathcal{P}_{n}\right) \mathcal{C}, \\
& A \mathbb{D} \mathcal{P}_{n}=\mathcal{B}_{n}^{+} \mathbb{E}^{+} \mathcal{P}_{n}-\left(\mathbb{E}^{-} \mathcal{P}_{n}\right) \mathcal{C},
\end{aligned}
$$

with the matrices $\mathcal{B}_{n}^{ \pm}$and $\mathcal{C}$ given by

$$
\mathcal{B}_{n}^{ \pm}=\left[\begin{array}{cc}
l_{n} \mp \Delta_{y} \pi_{n} & \Theta_{n} \\
-\frac{\Theta_{n-1}}{\gamma_{n}} & l_{n-1} \mp \Delta_{y} \pi_{n-1}+\frac{\Theta_{n-1}}{\gamma_{n}} \mathbb{E}^{ \pm}\left(x-\beta_{n}\right)
\end{array}\right], \quad \mathcal{C}=\left[\begin{array}{cc}
C / 2 & -D \\
0 & -C / 2
\end{array}\right] .
$$

From the compatibility of (29) and (30)-(31) we get the equations for the transfer matrices $\mathcal{A}_{n}$, for all $n \geq 1$ [7]:

$$
\begin{aligned}
& A \mathbb{D} \mathcal{A}_{n}=\mathcal{B}_{n}^{-} \mathbb{E}^{-} \mathcal{A}_{n}-\left(\mathbb{E}^{+} \mathcal{A}_{n}\right) \mathcal{B}_{n-1}^{-}, \\
& A \mathbb{D} \mathcal{A}_{n}=\mathcal{B}_{n}^{+} \mathbb{E}^{+} \mathcal{A}_{n}-\left(\mathbb{E}^{-} \mathcal{A}_{n}\right) \mathcal{B}_{n-1}^{+} .
\end{aligned}
$$

The compatibility conditions (32)-(33) yield the following relations for the polynomials $\pi_{n}, l_{n}, \Theta_{n}$, for all $n \geq 0[7,15]$ :

$$
\begin{aligned}
& \pi_{n+1}=-\frac{1}{2} \sum_{k=0}^{n+1} \frac{\Theta_{k-1}}{\gamma_{k}}, \\
& l_{n+1}+l_{n}+\mathbb{M}\left(x-\beta_{n+1}\right) \frac{\Theta_{n}}{\gamma_{n+1}}=0 \\
& -A+\mathbb{M}\left(x-\beta_{n+1}\right)\left(l_{n+1}-l_{n}\right)-\frac{\Delta_{y}^{2}}{2}\left(\pi_{n+1}+\pi_{n}\right)+\Theta_{n+1}=\frac{\gamma_{n+1}}{\gamma_{n}} \Theta_{n-1} .
\end{aligned}
$$


The following initial conditions hold:

$$
\begin{aligned}
& \pi_{-1}=0, \pi_{0}=-D / 2, \\
& \Theta_{-1}=D, \Theta_{0}=A-\frac{\Delta_{y}^{2}}{4} D-\left(l_{0}-C / 2\right) \mathbb{M}\left(x-\beta_{0}\right), \\
& l_{-1}=C / 2, l_{0}=-\mathbb{M}\left(x-\beta_{0}\right) D-C / 2 .
\end{aligned}
$$

3.3. Further matrix identities. The following results extend the differential systems from the continuous orthogonality given in [14] to the discrete orthogonality on systems of nonuniform lattices (see [3, Th. 1] and also [22, Sec. 4]). We stress equation (43) below, the analogue of the so-called Magnus' summation formula [14].

Theorem 1. Let $S$ be a Stieltjes function related to a weight $w$, satisfying $A \mathbb{D} S=C \mathbb{M} S+D$, and let $\left\{\mathcal{Y}_{n}\right\}_{n \geq 0}$ be the corresponding sequence given by $\left\{\mathcal{Y}_{n}=\left[\begin{array}{cc}P_{n+1} & q_{n+1} / w \\ P_{n} & q_{n} / w\end{array}\right]\right\}_{n \geq 0}$. The following equation holds:

$$
A_{n+1} \mathbb{D} \mathcal{Y}_{n}=\left(\mathcal{B}_{n}-C / 2 I\right) \mathbb{M} \mathcal{Y}_{n}, \quad n \geq 1,
$$

where

$$
A_{n+1}=A+\frac{\Delta_{y}^{2}}{2} \pi_{n}
$$

$I$ is the identity matrix, and $\mathcal{B}_{n}$ is given as

$$
\mathcal{B}_{n}=\left[\begin{array}{cc}
l_{n} & \Theta_{n} \\
-\frac{\Theta_{n-1}}{\gamma_{n}} & l_{n-1}+\frac{\Theta_{n-1}}{\gamma_{n}} \mathbb{M}\left(x-\beta_{n}\right)
\end{array}\right] .
$$

Corollary 1. The matrix $\mathcal{B}_{n}$ satisfies the following identities, for all $n \geq 1$ :

$$
\operatorname{tr} \mathcal{B}_{n}=0
$$

$$
\operatorname{det} \mathcal{B}_{n}=-\Delta_{y}^{2} \pi_{n}^{2}+A D-\frac{C^{2}}{4}+A \sum_{k=1}^{n} \frac{\Theta_{k-1}}{\gamma_{k}} .
$$

Remark . Taking into account $\Theta_{-1} / \gamma_{0}=D$ (see (38)) and (34), an equivalent equation for (43) is

$$
\operatorname{det} \mathcal{B}_{n}=-\Delta_{y}^{2} \pi_{n}^{2}-\frac{C^{2}}{4}-2 A \pi_{n} .
$$


In the account of (42), we shall use $\mathcal{B}_{n}$ in (44) given as

$$
\mathcal{B}_{n}=\left[\begin{array}{cc}
l_{n} & \Theta_{n} \\
-\Theta_{n-1} / \gamma_{n} & -l_{n}
\end{array}\right] .
$$

Therefore, (44) reads as

$$
-l_{n}^{2}(x)+\Theta_{n}(x) \frac{\Theta_{n-1}(x)}{\gamma_{n}}=-\Delta_{y}^{2} \pi_{n}^{2}-\frac{C^{2}}{4}-2 A \pi_{n} .
$$

\section{Difference equations for the recurrence relation coef- ficients}

4.1. Difference equations when $m=1$ in (21). Let us take $m=1$ in (21), that is, $A(x) \mathbb{D} S(x)=C(x) \mathbb{M} S(x)+D(x)$ with

$$
\operatorname{deg}(A) \leq 3, \operatorname{deg}(C) \leq 2, \operatorname{deg}(D) \leq 1,
$$

were we consider, by writing

$$
A(x)=a_{3} x^{3}+a_{2} x^{2}+a_{1} x+a_{0}, C(x)=c_{2} x^{2}+c_{1} x+c_{0},
$$

the condition

$$
a_{3} \neq 0 \text { or } c_{2} \neq 0 \text {. }
$$

The polynomial $D$ is given in terms of $A, C$. By collecting the coefficient of $x^{3}$ in (38) as well as the coefficient of $x^{2}$ in (39) we get

$$
d_{1}=-\left(a_{3}+c_{2} p_{1}\right) / p_{1}^{2} .
$$

By collecting the coefficient of $x^{2}$ in (38) as well as the coefficient of $x$ in (39) we get, using (48),

$$
d_{0}=\frac{a_{3}\left(2 p_{0} p_{1}-r_{1}-2 p_{1} \beta_{0}\right)-p_{1}\left(a_{2} p_{1}+c_{1} p_{1}^{2}+c_{2}\left(r_{1}+p_{1} \beta_{0}-p_{0} p_{1}\right)\right)}{p_{1}^{4}} .
$$

In the account of $(26)-(27)$, we have $\operatorname{deg}\left(l_{n}\right)=2, \operatorname{deg}\left(\Theta_{n}\right)=\operatorname{deg}\left(\pi_{n}\right)=1$. Set

$$
l_{n}(x)=\ell_{n, 2} x^{2}+\ell_{n, 1} x+\ell_{n, 0}, \Theta_{n}(x)=\Theta_{n, 1} x+\Theta_{n, 0}, \pi_{n}(x)=\pi_{n, 1} x+\pi_{n, 0} .
$$

Also, recall (8), thus $\Delta_{y}^{2}(x)=4 r(x)$.

Henceforth we adopt the convention that $\sum_{i}^{j} \cdot=0$ whenever $i>j$ and $\prod_{i}^{j} \cdot=1$ whenever $i>j$. 
Lemma 1. Under the previous assumptions and notations, the quantities $\ell_{n, 2}, \ell_{n, 1}, \Theta_{n, 1} / \gamma_{n+1}, \Theta_{n, 0} / \gamma_{n+1}, \pi_{n, 1}, \pi_{n, 0}$ are given, for all $n \geq 0$, by

$$
\begin{aligned}
\ell_{n, 2} & =n \frac{a_{3}}{p_{1}}-p_{1} d_{1}-\frac{c_{2}}{2}, \\
\frac{\Theta_{n, 1}}{\gamma_{n+1}} & =\frac{1}{p_{1}}\left(-(2 n+1) \frac{a_{3}}{p_{1}}+2 p_{1} d_{1}+c_{2}\right), \\
\pi_{n, 1} & =-\frac{d_{1}}{2}+\frac{1}{2 p_{1}}\left(\frac{a_{3}}{p_{1}} n-2 p_{1} d_{1}-c_{2}\right) n, \\
\ell_{n, 1} & =L_{n, 1}+\frac{a_{3}}{p_{1}^{2}} \sum_{k=1}^{n} \beta_{k} .
\end{aligned}
$$

with

$$
\begin{aligned}
& L_{n, 1}=\left(\frac{a_{2} p_{1}-a_{3} p_{0}}{p_{1}^{2}}\right) n \\
& +\frac{2 r_{1}}{p_{1}}\left(-n d_{1}+\frac{a_{3}}{2 p_{1}^{2}}\left(\frac{(n-1) n(2 n-1)}{3}+n^{2}\right)-\frac{\left(2 p_{1} d_{1}+c_{2}\right)}{2 p_{1}} n^{2}\right) \\
& -p_{1} d_{0}-\left(p_{0}-\beta_{0}\right) d_{1}-\frac{c_{1}}{2} \text {. }
\end{aligned}
$$

Furthermore, the following relations hold, for all $n \geq 1$ :

$$
\begin{array}{r}
\frac{\Theta_{n, 0}}{\gamma_{n+1}}=S_{n, 0}+\frac{1}{p_{1}}\left(\frac{\Theta_{n, 1}}{\gamma_{n+1}}-\frac{a_{3}}{p_{1}^{2}}\right) \beta_{n+1}-\frac{2 a_{3}}{p_{1}^{3}} \sum_{k=1}^{n} \beta_{k}, \\
S_{n, 0}=-\frac{\left(L_{n+1,1}+L_{n, 1}+p_{0} \Theta_{n, 1} / \gamma_{n+1}\right)}{p_{1}},
\end{array}
$$

(i) if $a_{3} \neq 0$, then

$$
\pi_{n, 0}=\hat{T}_{n, 0}+\frac{\ell_{n, 2}}{p_{1}^{2}} \sum_{k=1}^{n} \beta_{k}, \quad \hat{T}_{n, 0}=\frac{L_{n, 1} \ell_{n, 2}-2 r_{1} \pi_{n, 1}^{2}-a_{2} \pi_{n, 1}-c_{1} c_{2} / 4}{a_{3}}
$$


(ii) if $a_{3}=0$, then

$$
\begin{gathered}
\pi_{n, 0}=T_{n, 0}-\frac{\left(2 p_{1} d_{1}+c_{2}\right)}{2 p_{1}^{2}} \sum_{k=2}^{n} \beta_{k}, \\
T_{n, 0}=-\frac{d_{0}}{2}-\frac{1}{2} \frac{\Theta_{0,0}}{\gamma_{1}}+\frac{1}{2 p_{1}} \sum_{k=1}^{n-1}\left(L_{k+1,1}+L_{k, 1}+\frac{p_{0}\left(2 p_{1} d_{1}+c_{2}\right)}{p_{1}}\right) .
\end{gathered}
$$

The initial conditions hold:

$$
\begin{aligned}
\pi_{0,0} & =-\frac{d_{0}}{2} \\
\frac{\Theta_{0,0}}{\gamma_{1}} & =-\frac{a_{2}}{p_{1}^{2}}+\frac{r_{1}}{p_{1}^{2}}\left(d_{1}+\frac{\Theta_{0,1}}{\gamma_{1}}\right)+\left(-\ell_{0,2}-p_{1} \frac{\Theta_{0,1}}{\gamma_{1}}+\frac{c_{2}}{2}\right)\left(\frac{p_{0}-\beta_{1}}{p_{1}^{2}}\right) \\
& +\frac{1}{p_{1}}\left(-\ell_{0,1}-\left(p_{0}-\beta_{1}\right) \frac{\Theta_{0,1}}{\gamma_{1}}+\frac{c_{1}}{2}\right)+\frac{d_{1}}{p_{1}}\left(2 p_{0}-\left(\beta_{0}+\beta_{1}\right)+\frac{r_{1}}{p_{1}}\right)+d_{0} .
\end{aligned}
$$

Here, $p_{1}, p_{0}, r_{1}$ are the coefficients of $p(x), r(x)$, defined in (6).

Proof: The coefficient of $x^{3}$ in (36) yields

$$
-a_{3}+p_{1}\left(\ell_{n+1,2}-\ell_{n, 2}\right)=0 .
$$

This, combined with the initial condition $\ell_{0,2}=-p_{1} d_{1}-c_{2} / 2$, gives us (50).

The use of (50) in the equation that follows from the coefficient of $x^{2}$ in (35),

$$
\ell_{n+1,2}+\ell_{n, 2}+p_{1} \Theta_{n, 1} / \gamma_{n+1}=0
$$

gives us (51) for all $n \geq 1$. In order to get $\Theta_{0,1} / \gamma_{1}$ we take $n=1$ in

$$
A_{n+1} \mathbb{D} P_{n}^{(1)}=\left(l_{n}+C / 2\right) \mathbb{M} P_{n}^{(1)}+D \mathbb{M} P_{n+1}+\Theta_{n} \mathbb{M} P_{n-1}^{(1)} .
$$

Indeed, using (34) and (35) with $n=0$ in the equation above with $n=1$ we have

$$
A+2 r\left(-\frac{D}{2}-\frac{1}{2} \frac{\Theta_{0}}{\gamma_{1}}\right)=\left(-l_{0}-\mathbb{M}\left(x-\beta_{1}\right) \frac{\Theta_{0}}{\gamma_{1}}+C / 2\right) \mathbb{M}\left(x-\beta_{1}\right)+D \mathbb{M} P_{2}+\Theta_{1} .
$$

The coefficient of $x^{3}$ gives us

$$
\frac{\Theta_{0,1}}{\gamma_{1}}=\frac{1}{p_{1}}\left(-\frac{a_{3}}{p_{1}}+2 p_{1} d_{1}+c_{2}\right)
$$

thus (51) also holds for $n=0$. 
Equation (52) follows from the use of (51) in the summation formula (34), and the initial condition $\pi_{0,1}=-d_{1} / 2$ (cf. (37)).

Let us now obtain (53). Using (50) in the equation that follows from equating the coefficients of $x^{2}$ in (36) we get

$$
\ell_{n+1,1}=\ell_{n, 1}-\frac{\left(p_{0}-\beta_{n+1}\right) a_{3}}{p_{1}^{2}}+\frac{2 r_{1}}{p_{1}} \lambda_{n, 1}+\frac{a_{2}}{p_{1}}, \quad \lambda_{n, 1}=\pi_{n+1,1}+\pi_{n, 1} .
$$

Thus, we obtain (53), where we used the initial conditions $\ell_{0,1}=-p_{1} d_{0}-$ $\left(p_{0}-\beta_{0}\right) d_{1}-c_{1} / 2$.

To get (54) we take the $x$ coefficient in (35),

$$
\ell_{n+1,1}+\ell_{n, 1}+p_{1} \frac{\Theta_{n, 0}}{\gamma_{n+1}}+\left(p_{0}-\beta_{n+1}\right) \frac{\Theta_{n, 1}}{\gamma_{n+1}}=0,
$$

and substitute (53) and (51) therein. The initial condition $\pi_{0,0}$ comes from (37) and $\Theta_{0,0} / \gamma_{1}$ follows from taking the coefficient of $x^{2}$ in (57).

$\pi_{n, 0}$ can be obtained via the summation formula (34). Thus, from (34), if $a_{3}=0$, we get

$$
\pi_{n, 0}=-\frac{1}{2} \frac{\Theta_{-1,0}}{\gamma_{0}}-\frac{1}{2} \frac{\Theta_{0,0}}{\gamma_{1}}-\frac{1}{2} \sum_{k=1}^{n-1} \frac{\Theta_{k, 0}}{\gamma_{k+1}} .
$$

and (56) follows. The case $a_{3} \neq 0$ can be alternatively obtained through the use of the $x^{3}$-coefficient in (45), thus yielding (55).

Theorem 2. Let $S$ be a Stieltjes function satisfying

$$
A(x) \mathbb{D} S(x)=C(x) \mathbb{M} S(x)+D(x)
$$

with $\operatorname{deg}(A) \leq 3, \operatorname{deg}(C) \leq 2, \operatorname{deg}(D) \leq 1$ subject to the condition (47). Let $\left\{P_{n}\right\}_{n \geq 0}$ be the corresponding SMOP, satisfying (13),

$$
P_{n+1}(x)=\left(x-\beta_{n}\right) P_{n}(x)-\gamma_{n} P_{n-1}(x), \quad n=0,1,2, \ldots .
$$

Under the notations of the previous lemma, the $\gamma_{n}$ 's are defined only in terms of the $\beta_{n}$ 's and the polynomials $A, C$, a well as $p, r$ from (6), related to the quadratic lattice. There holds the formula

$$
\begin{aligned}
\gamma_{n+2} & =\gamma_{1} \prod_{j=0}^{n} s_{j}+\sum_{k=0}^{n} t_{k} \prod_{j=k+1}^{n} s_{j}, \quad n \geq 0, \\
\gamma_{1} & =\frac{p_{1} \Theta_{0,1}}{-a_{3} / p_{1}+2 p_{1} d_{1}+c_{2}},
\end{aligned}
$$


with

$$
\begin{gathered}
s_{n}=\frac{\Theta_{n-1} / \gamma_{n}}{\Theta_{n+1} / \gamma_{n+2}}\left(x_{n+1}\right), \quad t_{n}=\frac{A\left(x_{n+1}\right)+2 r\left(x_{n+1}\right)\left(\pi_{n+1}+\pi_{n}\right)\left(x_{n+1}\right)}{\left(\Theta_{n+1} / \gamma_{n+2}\right)\left(x_{n+1}\right)}, \\
x_{n+1}=\left(\beta_{n+1}-p_{0}\right) / p_{1},
\end{gathered}
$$

and

$\Theta_{0,1}=a_{1}-r_{1} d_{0}-r_{0} d_{1}+\left(p_{1} d_{0}+\left(p_{0}-\beta_{0}\right) d_{1}+c_{1}\right)\left(p_{0}-\beta_{0}\right)+\left(\left(p_{0}-\beta_{0}\right) d_{0}+c_{0}\right) p_{1}$.

Proof: We evaluate $(36)$ at $x_{n+1}=\left(\beta_{n+1}-p_{0}\right) / p_{1}$. As $\mathbb{M}\left(x-\beta_{n+1}\right)\left(x_{n+1}\right)=0$, we get

$$
-A\left(x_{n+1}\right)-2 r\left(x_{n+1}\right) \lambda_{n}\left(x_{n+1}\right)+\gamma_{n+2} \frac{\Theta_{n+1}}{\gamma_{n+2}}\left(x_{n+1}\right)=\gamma_{n+1} \frac{\Theta_{n-1}}{\gamma_{n}}\left(x_{n+1}\right),
$$

where it was used the notation $\lambda_{n}(x)=\pi_{n+1}(x)+\pi_{n}(x)$. Hence, we obtain

$$
\gamma_{n+2}=s_{n} \gamma_{n+1}+t_{n}, \quad n \geq 0
$$

with $s_{n}, t_{n}$ given in (63). As the solution of the initial value problem

$$
z_{n+1}=a_{n} z_{n}+b_{n}, \quad z_{n_{0}}=z_{0}
$$

is $[6]$

$$
z_{n}=z_{0} \prod_{j=n_{0}}^{n-1} a_{j}+\sum_{k=n_{0}}^{n-1} b_{k} \prod_{j=k+1}^{n-1} a_{j},
$$

then equation (61) is a consequence of (66).

To get $\gamma_{1}$, we take the initial conditions

$$
\begin{aligned}
\ell_{0,1} & =-p_{1} d_{0}-\left(p_{0}-\beta_{0}\right) d_{1}-c_{1} / 2, \\
\ell_{0,0} & =-\left(p_{0}-\beta_{0}\right) d_{0}-c_{0} / 2 . \\
\Theta_{0,1} & =a_{1}-r_{1} d_{0}-r_{0} d_{1}-\left(\ell_{0,1}-c_{1} / 2\right)\left(p_{0}-\beta_{0}\right)-\left(\ell_{0,0}-c_{0} / 2\right) p_{1},
\end{aligned}
$$

The use of (67) and (68) in (69) yields (64). Using $\Theta_{0,1} / \gamma_{1}$ given by (cf. (58))

$$
\frac{\Theta_{0,1}}{\gamma_{1}}=\frac{1}{p_{1}}\left(-a_{3} / p_{1}+2 p_{1} d_{1}+c_{2}\right)
$$

combined with (64), we get (62). 
Remark. The previous theorem gives us the $\gamma_{n}$ 's in terms of the $\beta_{n}$ 's. In order to obtain a recurrence for the $\beta_{n}$ 's we may start by taking the independent term of (35), which gives us

$$
\ell_{n+1,0}=-\ell_{n, 0}-\left(p_{0}-\beta_{n+1}\right) \frac{\Theta_{n, 0}}{\gamma_{n+1}} .
$$

Using this equality into the equation that results from the coefficient of $x$ in (36) we obtain

$$
\begin{aligned}
\ell_{n, 0}=-\frac{1}{2 p_{1}}\left(a_{1}+2 r_{0} \lambda_{n, 1}\right) & -\frac{\left(p_{0}-\beta_{n+1}\right)}{2 p_{1}}\left(p_{1} \frac{\Theta_{n, 0}}{\gamma_{n+1}}+\ell_{n, 1}-\ell_{n+1,1}\right) \\
& -\frac{r_{1}}{p_{1}}\left(\pi_{n+1,0}+\pi_{n, 0}\right)+\gamma_{n+2} \frac{\nu_{n+1,1}}{2 p_{1}}-\gamma_{n+1} \frac{\nu_{n-1,1}}{2 p_{1}},
\end{aligned}
$$

where we used the notation $\lambda_{n, 1}=\pi_{n+1,1}+\pi_{n, 1}, \nu_{n, 1}=\Theta_{n, 1} / \gamma_{n+1}$. Now, by substituting (61) and (71) into equation (70) we get a first order non-linear recurrence relation for the $\beta_{n}$ 's, say $F\left(\gamma_{1}, \beta_{1}, \ldots, \beta_{n+2}\right)=G\left(\gamma_{1}, \beta_{1}, \ldots, \beta_{n+1}\right)$. Due to the complexity of such a formulae, we shall not give its explicit form here.

4.1.1. The symmetric case. The symmetric case, that is, $\beta_{n}=0, n \geq$ 0 , implies simplifications in (53), (54), (55), (56), and all these quantities now depend only on the lattice as well as on the coefficients $A, C, D$ of the difference equation for the Stieltjes function. In such a case, we have the result that follows.

Corollary 2. Let $A(x) \mathbb{D} S(x)=C(x) \mathbb{M} S(x)+D(x)$ with $\operatorname{deg}(A) \leq 3$, $\operatorname{deg}(C) \leq 2, \operatorname{deg}(D) \leq 1$ subject to the condition (47). Under the previous notation, let $\beta_{n}=0, n \geq 0$. Then, the $\gamma_{n}$ 's are determined through:

$$
\begin{aligned}
\gamma_{n+2} & =\gamma_{1} \prod_{j=0}^{n} s_{j}+\sum_{k=0}^{n} t_{k} \prod_{j=k+1}^{n} s_{j}, \quad n \geq 0 \\
\gamma_{1} & =\frac{p_{1} \Theta_{0,1}}{-a_{3} / p_{1}+2 p_{1} d_{1}+c_{2}}
\end{aligned}
$$

with

$s_{n}=\frac{\left(\Theta_{n-1} / \gamma_{n}\right)\left(x_{0}\right)}{\left(\Theta_{n+1} / \gamma_{n+2}\right)\left(x_{0}\right)}, \quad t_{n}=\frac{A\left(x_{0}\right)+2 r\left(x_{0}\right)\left(\pi_{n+1}+\pi_{n}\right)\left(x_{0}\right)}{\left(\Theta_{n+1} / \gamma_{n+2}\right)\left(x_{0}\right)}, x_{0}=-p_{0} / p_{1}$,

and

$$
\Theta_{0,1}=a_{1}-r_{1} d_{0}-r_{0} d_{1}+\left(p_{1} d_{0}+p_{0} d_{1}+c_{1}\right) p_{0}+\left(p_{0} d_{0}+c_{0}\right) p_{1} .
$$


Proof: Take $\beta_{n}=0, n \geq 0$. Evaluate (36) at $x_{0}=-p_{0} / p_{1}$. Thus, as $\mathbb{M}\left(x_{0}\right)=$ 0 , we get

$$
-A\left(x_{0}\right)-2 r\left(x_{0}\right)\left(\pi_{n+1}+\pi_{n}\right)\left(x_{0}\right)+\gamma_{n+2} \frac{\Theta_{n+1}}{\gamma_{n+2}}\left(x_{0}\right)=\gamma_{n+1} \frac{\Theta_{n-1}}{\gamma_{n}}\left(x_{0}\right) .
$$

Therefore, (72) follows.

4.1.2. Condition (47) with $a_{3}=0$. Let us take the case

$$
\operatorname{deg}(A) \leq 2, \operatorname{deg}(C)=2, \operatorname{deg}(D)=1 .
$$

In such a case, the quantities given in Lemma 1 are as follow:

$$
\begin{aligned}
\ell_{n, 2} & =\frac{c_{2}}{2}, \quad \ell_{n, 1}=L_{n, 1}, \\
\frac{\Theta_{n, 1}}{\gamma_{n+1}} & =-\frac{c_{2}}{p_{1}}, \frac{\Theta_{n, 0}}{\gamma_{n+1}}=S_{n, 0}-\frac{c_{2}}{p_{1}^{2}} \beta_{n+1}, \\
\pi_{n, 1} & =(n+1) \frac{c_{2}}{2 p_{1}}, \quad \pi_{n, 0}=T_{n, 0}+\frac{c_{2}}{2 p_{1}^{2}} \sum_{k=2}^{n} \beta_{k},
\end{aligned}
$$

with

$$
\begin{aligned}
& L_{n, 1}=\frac{n a_{2}}{p_{1}}+\frac{2 r_{1}}{p_{1}} \frac{n c_{2}}{p_{1}}\left(1+\frac{n}{2}\right)-p_{1} d_{0}-\left(p_{0}-\beta_{0}\right) d_{1}-\frac{c_{1}}{2}, \\
& S_{n, 0}=-\frac{\left(L_{n+1,1}+L_{n, 1}+p_{0} \Theta_{n, 1} / \gamma_{n+1}\right)}{p_{1}}, \\
& T_{n, 0}=-\frac{d_{0}}{2}-\frac{1}{2} \frac{\Theta_{0,0}}{\gamma_{1}}+\frac{1}{2 p_{1}} \sum_{k=1}^{n-1}\left(L_{k+1,1}+L_{k, 1}-\frac{c_{2} p_{0}}{p_{1}}\right) .
\end{aligned}
$$

Furthermore, by taking the $x^{2}$-coefficient of (44), that is,

$$
\begin{aligned}
-\ell_{n, 1}^{2}-2 \ell_{n, 2} \ell_{n, 0}+\gamma_{n+1} & \frac{\Theta_{n, 1}}{\gamma_{n+1}} \frac{\Theta_{n-1,1}}{\gamma_{n+1}}=-8 r_{1} \pi_{n, 0} \pi_{n, 1} \\
& -4 r_{0} \pi_{n, 1}^{2}-\left(c_{1}^{2}+2 c_{2} c_{0}\right) / 4-2 a_{2} \pi_{n, 0}-2 a_{1} \pi_{n, 1},
\end{aligned}
$$

we get the expression for $\ell_{n, 0}$,

$$
\ell_{n, 0}=\tau_{n}+\frac{c_{2}}{p_{1}^{2}} \gamma_{n+1}+\left(4 r_{1}(n+1)+2 \frac{a_{0}}{c_{2}}\right) \pi_{n, 0},
$$

with

$$
\tau_{n}=-\frac{\ell_{n, 1}^{2}}{c_{2}}+r_{0} \frac{c_{2}}{p_{1}^{2}}(n+1)^{2}+\frac{\left(c_{1}^{2}+2 c_{2} c_{0}\right)}{4 c_{2}} .
$$


Theorem 3. Under the degrees (75) and the previous notations, we have the following difference equations for the recurrence coefficients, for all $n \geq 1$ :

$$
\begin{gathered}
\gamma_{n+2}+\gamma_{n+1}+\frac{p_{1}}{c_{2}}\left(4 r_{1}(n+2)+2 \frac{a_{0}}{c_{2}}\right) \pi_{n+1,0}+\frac{p_{1}}{c_{2}}\left(4 r_{1}(n+1)+2 \frac{a_{0}}{c_{2}}\right) \pi_{n, 0} \\
+\frac{p_{1}}{c_{2}}\left(p_{0}-\beta_{n+1}\right) \frac{\Theta_{n, 0}}{\gamma_{n+1}}+\frac{p_{1}}{c_{2}}\left(\tau_{n+1}+\tau_{n}\right)=0 \\
\gamma_{n+1}=\frac{2 L_{n, 1}\left(\tau_{n}+\left(4 r_{1}(n+1)+2 a_{0} / c_{2}\right) \pi_{n, 0}\right)+G_{n}}{\frac{-2 c_{2}}{p_{1}^{2}} L_{n, 1}-\frac{c_{2}}{p_{1}}\left(\frac{\Theta_{n-1,0}}{\gamma_{n}}+\frac{\Theta_{n, 0}}{\gamma_{n+1}}\right)}
\end{gathered}
$$

with the initial condition $\gamma_{1}$ given by (62), and with $G_{n}=-4 r_{1} \pi_{n, 0}^{2}-8 r_{0} \pi_{n, 0} \pi_{n, 1}-$ $c_{1} c_{0} / 2-2 a_{1} \pi_{n, 0}-2 a_{0} \pi_{n, 1}$.

Proof: To get (78) we use (77) in the equation obtained from the independent term in $(35)$,

$$
\ell_{n+1,0}+\ell_{n, 0}+\left(p_{0}-\beta_{n+1}\right) \frac{\Theta_{n, 0}}{\gamma_{n+1}}=0 .
$$

To get (79) we take the $x$-coefficient of (44), that is,

$$
-2 \ell_{n, 1} \ell_{n, 0}+\gamma_{n+1}\left(\frac{\Theta_{n, 1}}{\gamma_{n+1}} \frac{\Theta_{n-1,0}}{\gamma_{n}}+\frac{\Theta_{n, 0}}{\gamma_{n+1}} \frac{\Theta_{n-1,1}}{\gamma_{n}}\right)=G_{n}
$$

with $G_{n}=-4 r_{1} \pi_{n, 0}^{2}-8 r_{0} \pi_{n, 0} \pi_{n, 1}-c_{1} c_{0} / 2-2 a_{1} \pi_{n, 0}-2 a_{0} \pi_{n, 1}$. The use of $\ell_{n, 0}$ given by (77) into the above equation gives us (79).

\section{2. $m=0$ in (21): classical orthogonal polynomials on quadratic} lattices from compatibility relations. Let us take $m=0$ in (21), that is, $A(x) \mathbb{D} S(x)=C(x) \mathbb{M} S(x)+D(x)$ with

$$
\operatorname{deg}(A) \leq 2, \operatorname{deg}(C) \leq 1, \operatorname{deg}(D)=0 .
$$

were we consider, by writing

$$
A(x)=a_{2} x^{2}+a_{1} x+a_{0}, C(x)=c_{1} x+c_{0},
$$

the condition

$$
a_{2} \neq 0 \text { or } c_{1} \neq 0 \text {. }
$$

In the account of $(26)-(27)$, we have $\operatorname{deg}\left(l_{n}\right)=1, \operatorname{deg}\left(\Theta_{n}\right)=\operatorname{deg}\left(\pi_{n}\right)=0$. Set

$$
l_{n}(x)=\ell_{n, 1} x+\ell_{n, 0}, \Theta_{n}(x)=\Theta_{n, 0}, \pi_{n}(x)=\pi_{n, 0} .
$$

We have $D=d_{0}=-\left(a_{2}+c_{1} p_{1}\right) / p_{1}^{2}$. 
Lemma 2. Under the previous assumptions and notations, we have, for all $n \geq 0$,

$$
\begin{aligned}
\ell_{n, 1} & =n \frac{a_{2}}{p_{1}}-p_{1} d_{0}-\frac{c_{1}}{2} \\
\frac{\Theta_{n, 0}}{\gamma_{n+1}} & =\frac{1}{p_{1}}\left(-(2 n+1) \frac{a_{2}}{p_{1}}+2 p_{1} d_{0}+c_{1}\right), \\
\pi_{n, 0} & =-\frac{d_{0}}{2}+\frac{1}{2 p_{1}}\left(\frac{a_{2}}{p_{1}} n-2 p_{1} d_{0}-c_{1}\right) n
\end{aligned}
$$

and

$$
\ell_{n, 0}=\frac{2 r_{1} \pi_{n, 0}^{2}+c_{0} c_{1} / 4+a_{1} \pi_{n, 0}}{\ell_{n, 1}}, n \geq 1, \quad \ell_{0,0}=-\left(p_{0}-\beta_{0}\right) d_{0}-c_{0} / 2 .
$$

Here, $p_{1}, p_{0}, r_{1}$ are the coefficients of $p(x), r(x)$, defined in (6).

Proof: The equations (82)-(84) follow from Lemma 1.

The $x$-coefficient of (45) gives us (85).

Theorem 4. Let $A(x) \mathbb{D} S(x)=C(x) \mathbb{M} S(x)+D(x)$ with $\operatorname{deg}(A) \leq 2, \operatorname{deg}(C)$ $\leq 1, \operatorname{deg}(D) \leq 0$ subject to the condition (81). Consider the notations of the previous lemma. The following holds:

$$
\begin{aligned}
& \gamma_{n+1}=\frac{\ell_{n, 0}^{2}-4 r_{0} \pi_{n, 0}^{2}-c_{0}^{2} / 4-2 a_{0} \pi_{n, 0}}{\frac{\Theta_{n, 0}}{\gamma_{n+1}} \frac{\Theta_{n-1,0}}{\gamma_{n}}}, \quad n \geq 1, \\
& \beta_{n+1}=\frac{\ell_{n+1,0}+\ell_{n, 0}+p_{0} \Theta_{n, 0} / \gamma_{n+1}}{\Theta_{n, 0} / \gamma_{n+1}}, \quad n \geq 0,
\end{aligned}
$$

and the initial conditions $\beta_{0}$ and $\gamma_{1}$ given by

$$
\begin{aligned}
\beta_{0} & =\frac{p_{0} d_{0}+c_{0}+\left(a_{1}-r_{1} d_{0}\right) / p_{1}-a_{2} p_{0} / p_{1}^{2}}{d_{0}-a_{2} / p_{1}^{2}}, \\
\gamma_{1} & =\frac{p_{1}\left(a_{0}-r_{0} d_{0}+\left(\left(p_{0}-\beta_{0}\right) d_{0}+c_{0}\right)\left(p_{0}-\beta_{0}\right)\right)}{-a_{2} / p_{1}+2 p_{1} d_{0}+c_{1}} .
\end{aligned}
$$

Proof: The equation (86) follows from the independent coefficient of (45),

$$
-\ell_{n, 0}^{2}+\gamma_{n+1} \frac{\Theta_{n, 0}}{\gamma_{n+1}} \frac{\Theta_{n-1,0}}{\gamma_{n}}=-4 r_{0} \pi_{n, 0}^{2}-c_{0}^{2} / 4-2 a_{0} \pi_{n, 0} .
$$


The equation (87) is obtained from the independent term of (35),

$$
\ell_{n+1,0}+\ell_{n, 0}+\left(p_{0}-\beta_{n+1}\right) \frac{\Theta_{n, 0}}{\gamma_{n+1}}=0 .
$$

To obtain $\beta_{0}$ and $\gamma_{1}$ we equate coefficients in (38) and (39), thus getting

$$
\begin{aligned}
\ell_{0,1} & =-p_{1} d_{0}-c_{1} / 2, \\
\ell_{0,0} & =-\left(p_{0}-\beta_{0}\right) d_{0}-c_{0} / 2 . \\
0 & =a_{1}-r_{1} d_{0}-\left(\ell_{0,1}-c_{1} / 2\right)\left(p_{0}-\beta_{0}\right)-\left(\ell_{0,0}-c_{0} / 2\right) p_{1}, \\
\Theta_{0,0} & =a_{0}-r_{0} d_{0}-\left(\ell_{0,0}-c_{0} / 2\right)\left(p_{0}-\beta_{0}\right) .
\end{aligned}
$$

The use of (90) and (91) in (92) yields $\beta_{0}$. From (93) we have, using (91),

$$
\Theta_{0,0}=a_{0}-r_{0} d_{0}+\left(\left(p_{0}-\beta_{0}\right) d_{0}+c_{0}\right)\left(p_{0}-\beta_{0}\right) .
$$

From $\Theta_{0,0} / \gamma_{1}$ given by

$$
\frac{\Theta_{0,0}}{\gamma_{1}}=\frac{1}{p_{1}}\left(-a_{2} / p_{1}+2 p_{1} d_{0}+c_{1}\right)
$$

combined with (94) we get $\gamma_{1}$.

\section{Examples}

5.1. Dual Hahn polynomials. The Dual Hahn polynomials have the hypergeometric representation [13]

$$
P_{n}(x ; \gamma, \delta, N)={ }_{3} F_{2}\left(\begin{array}{c}
-n, n+\gamma+\delta+1,-x \\
\gamma+1,-N
\end{array} ; 1\right) .
$$

The lattice $x(s)$ and the polynomials $p, r$ that follow from (7) are

$$
x(s)=s(s+\gamma+\delta+1), p(x)=x+\frac{1}{4}, r(x)=x+\frac{(\gamma+\delta+1)^{2}}{4} .
$$

$\left\{P_{n}\right\}_{n \geq 0}$ is related to a linear functional $L$ that satisfies $\mathbb{D}(\phi L)=\mathbb{M}(\psi L)$, where the polynomials $\phi, \psi$ are given by [10] $\phi(x)=(-1+2 N+\delta-\gamma) x+N(1+\gamma)(1+\gamma+\delta), \quad \psi(x)=-2 x+2 N(1+\gamma)$.

The Stieltjes function satisfies (18), $A \mathbb{D} S=C \mathbb{M} S+D$, with $A, C$ given by (20), thus,

$$
A=\mathbb{M} \phi+2 r(x)-\frac{1}{2} \mathbb{M} \psi, \quad C=-1-\mathbb{D} \phi+\mathbb{M} \psi
$$


where we used $U_{1}=1 / 2$. The polynomial $D$ is a constant, $D=-c_{1} / p_{1}$. As we have $\operatorname{deg}(A)=\operatorname{deg}(C)=1$, condition (81) of Section 4.2 holds.

From the formulae in Theorem 4 we recover [13, pp. 209], for all $n \geq 1$, $\beta_{n}=(n+\gamma+1)(n-N)+n(n-\delta-N-1), \quad \gamma_{n}=n(n+\gamma)(n-1-N)(n-\delta-N-1)$, and $\beta_{0}=-N(\gamma+1), \gamma_{0}=1$.

5.2. Modification of Dual Hahn polynomials. We consider the following modifiation of the Dual Hahn polynomials. We take the linear functional [10, Sec. 2.4]

$$
\tilde{L}=\left(x+\frac{(\gamma+\delta+1)^{2}}{4}\right) L
$$

being $L$ the linear functional related to the Dual Hahn polynomials. $\tilde{L}$ satisfies

$$
\mathbb{D}(\tilde{\phi} \tilde{L})=\mathbb{M}(\tilde{\psi} \tilde{L}),
$$

where the polynomials $\tilde{\phi}, \tilde{\psi}$ are given by (see [10, Eq. (40)])

$$
\tilde{\phi}(x)=(r(x)+1) \phi(x)+2 r(x) \psi(x), \quad \tilde{\psi}(x)=(r(x)+1) \psi(x)+2 \phi(x),
$$

with $\phi, \psi$ given in (97). Note that (96) holds. Recall that we are taking $\alpha=1$ and $x-c=r(x)$, with our notation $r(x)$ for the polynomial $U_{2}(x)$, in [10, Eq. (40)].

Denote by $\left\{\tilde{P}_{n}\right\}_{n \geq 0}$ the SMOP related to $\tilde{L}$, and its recurrence relation coefficients by $\tilde{\beta}_{n}, \tilde{\gamma}_{n}$. The corresponding Stieltjes function satisfies (18), $\tilde{A} \mathbb{D} S=\tilde{C} \mathbb{M} S+\tilde{D}$, with $\tilde{A}, \tilde{C}$ given by (20), thus,

$$
\tilde{A}=\mathbb{M} \tilde{\phi}-r \mathbb{D} \tilde{\psi}-\frac{1}{2} \mathbb{M} \tilde{\psi}, \quad \tilde{C}=-\mathbb{D} \tilde{\phi}+\mathbb{M} \tilde{\psi}+\frac{1}{2} \mathbb{D} \tilde{\psi} .
$$

$\tilde{D}$ is a polynomial of degree one, with coefficients given by (48) and (49). As we have $\operatorname{deg}(A)=\operatorname{deg}(C)=2$, condition (75) of Sub-Section 4.1.2 holds. From Theorem 3 , the coefficients $\tilde{\gamma}_{n}, \tilde{\beta}_{n}$ are governed through the difference system (78)-(79).

Remark . The modification (99) is related to the Christoffel transformation [23, Sec. 3]. In this case the modified recurrence relation coefficients are known to be given in terms of the non-modified ones [23],

$\tilde{\beta}_{n}=\beta_{n+1}-\frac{P_{n+1}(c)}{P_{n}(c)}+\frac{P_{n+2}(c)}{P_{n+1}(c)}, \tilde{\gamma}_{n}=\gamma_{n} \frac{P_{n-1}(c) P_{n+1}(c)}{P_{n}^{2}(c)}, c=-\frac{(\gamma+\delta+1)^{2}}{4}$. 
Note that here the $P_{n}$ 's at $c$ must be evaluated trough (95), whilst our formulae in Theorem 3 give a relation for $\tilde{\beta}_{n}, \tilde{\gamma}_{n}$ in terms of the lattice and the polynomials involved in the difference equation for $\tilde{S}$.

\section{References}

[1] R. Askey and J. Wilson, Some basic hypergeometric orthogonal polynomials that generalize Jacobi polynomials, Memoirs AMS vol. 54 n. 319, AMS, Providence, 1985.

[2] S. Belmehdi, On semi-classical linear functionals of class $s=1$. Classification and integral representations, Indag Math. 3 (1992), pp. 253-275.

[3] A. Branquinho, Y. Chen, G. Filipuk, and M.N. Rebocho, A characterization theorem for semiclassical orthogonal polynomials on non uniform lattices, Appl. Math. Comput. 334 (2018), pp. 356-366.

[4] A. Branquinho and M.N. Rebocho, Characterization theorem for Laguerre-Hahn orthogonal polynomials on non-uniform lattices, J. Math. Anal. Appl. 427 (2015), pp. 185-201.

[5] D. Dominici and F. Marcellán, Discrete semiclassical orthogonal polynomials of class one, Pacific J. Math. 268 (2014), pp. 389-411.

[6] S. Elaydi, An introduction to difference equations. Undergraduate texts in Mathematics. Springer, New York, third ed. (2005).

[7] G. Filipuk and M.N. Rebocho, Orthogonal polynomials on systems of non-uniform lattices from compatibility conditions, J. Math. Anal. Appl. 456 (2017), pp. 1380-1396.

[8] M. Foupouagnigni, On difference equations for orthogonal polynomials on nonuniform lattices, J. Difference Equ. Appl. 14 (2008), pp. 127-174.

[9] M. Foupouagnigni, M. Kenfack Nangho, and S. Mboutngam, Characterization theorem for classical orthogonal polynomials on non-uniform lattices: the functional approach, Integral Transforms Spec. Funct. 22 (2011), pp. 739-758.

[10] S. Mboutngama, M. Foupouagnigni, and P. Njionou Sadjang, On the modifications of semiclassical orthogonal polynomials on nonuniform lattices, J. Math. Anal. Appl. 445 (2017), pp. 819-836.

[11] M. Njinkeu Sandjon, A. Branquinho, M. Foupouagnigni, and I. Area, Characterizations of classical orthogonal polynomials on quadratic lattices, J. Difference Equ. Appl. 23 (2017), pp. 983-1002

[12] M.E.H. Ismail, Classical and Quantum Orthogonal Polynomials in One Variable, vol. 98 of Encyclopedia of Mathematics and its Applications, Cambridge University Press, Cambridge, 2005.

[13] R. Koekoek and R. Swarttouw, The Askey-scheme of hypergeometric orthogonal polynomials and its q-analogue, Faculty of Information Technology and Systems, Delft University of Technology, Netherlands, Report no. 98-17, 1998.

[14] A.P. Magnus, Painlevé-type differential equations for the recurrence coefficients of semiclassical orthogonal polynomials, J. Comput. Appl. Math. 57 (1995), pp. 215-237.

[15] A.P. Magnus, Associated Askey-Wilson polynomials as Laguerre-Hahn orthogonal polynomials, Springer Lect. Notes in Math. 1329, Springer, Berlin, 1988, pp. 261-278.

[16] A.P. Magnus, Special nonuniform lattice (snul) orthogonal polynomials on discrete dense sets of points, J. Comput. Appl. Math. 65 (1995), pp. 253-265.

[17] P. Maroni and M. Mejri, The symmetric $D_{\omega}$-semiclassical orthogonal polynomials of class one, Numer. Algorithms, 49 (2008), pp. 251-282.

[18] A.F. Nikiforov, S.K. Suslov, Classical Orthogonal Polynomials of a discrete variable on non uniform lattices, Letters Math. Phys. 11 (1986), pp. 27-34. 
[19] A.F. Nikiforov, S.K. Suslov, and V.B. Uvarov, Classical Orthogonal Polynomials of a Discrete Variable. Springer, Berlin, 1991.

[20] S.K. Suslov, On the theory of difference analogues of special functions of hypergeometric type, Usp. Mat. Nauk 4 (1989), pp. 185-226.

[21] G. Szegő, Orthogonal Polynomials, Amer. Math. Soc. Colloq. Publ. 23, Amer. Math. Soc. Providence, RI, 1975 (Fourth Edition).

[22] N.S. Witte, Semi-classical orthogonal polynomial systems on nonuniform lattices, deformations of the Askey table, and analogues of isomonodromy, Nagoya Math. J. 219 (2015), pp. 127-234.

[23] A. Zhedanov, Rational spectral transformations and orthogonal polynomials, J. Comput. Appl. Math. 85 (1997), pp. 67-86.

G. FILIPUK

Faculty of Mathematics, Informatics and Mechanics, University of Warsaw, Banacha 2, WARSAW, 02-097, POLAND.

E-mail address: filipuk@mimuw.edu.pl

M.N. REBOCHO

Departamento de Matemática, Universidade da Beira interior, 6201-001 Covilhã, Portugal; CMUC, Department of Mathematics, University of Coimbra, 3001-501 Coimbra, PORTUGAL.

E-mail address: mneves@ubi.pt 